\title{
Intramedullary abscess complicating congenital dermal sinus tract in an infant: A rare occurrence
}

\author{
*Joseph J Valamparampil ${ }^{1,}$ Ajay Kumar², Alice Deepa ${ }^{3}$, Prameela Joji ${ }^{4}$, S Shajehan ${ }^{5}$
}

Sri Lanka Journal of Child Health, 2017; 46(1): 77-79

DOI: http://dx.doi.org/10.4038/sljch.v46i1.7984

(Keywords: Intramedullary spinal cord abscess, congenital dermal sinus, methicillin-resistant Staphylococcus aureus, coccygeal pit, magnetic resonance imaging)

\section{Case report}

A four month old baby girl was brought to our department with a history of progressive decrease in movements of the left lower limb of three weeks duration and right lower limb of one day duration. The baby was intermittently febrile since three days prior to hospital admission and also had retention of urine of one day duration. The baby did not have any seizures, altered sensorium, vomiting or any history suggestive of central nervous system (CNS) involvement or raised intracranial tension. Parents noticed decreased sensations in lower limbs. The mother also reported that when pinched on the left leg she was not wincing or crying. She had a birth mark with a dimple in the lower back from which a recurrent discharge of white fluid was present since one and a half months of age. The parents had consulted multiple doctors including a neurosurgeon for the same but was reassured and sent back.

On examination at the time of admission, the baby was conscious and alert, but irritable. The cranial nerves and upper limbs were normal. She had dorsolumbar scoliosis with convexity to the left, lax anterior abdominal wall on the left side and flaccid anal sphincter. There was a pale pink patch $(3 \times 3 \mathrm{~cm})$ with a central dimple in the lumbosacral area, approximately $3.5 \mathrm{~cm}$ above the gluteal cleft suggestive of a congenital dermal sinus with neuro-

${ }^{1}$ Senior Registrar, ${ }^{2}$ Resident, Department of
Paediatrics, ${ }^{3}$ Resident, Department
Radiodiagnosis, ${ }^{4}$ Consultant, Department of
Paediatrics, ${ }^{5}$ Senior Consultant, Department of
Neurosurgery, Kerala Institute of Medical
Sciences, India
${ }^{*}$ Correspondence: josephvalam@yahoo.co.in

(Received on 05 June 2015: Accepted after revision on 17 July 2015)

The authors declare that there are no conflicts of interest

Personal funding was used for the project.

Open Access Article published under the Creative cutaneous marker (Figure 1). Her lower limbs were hypotonic with complete paralysis (power 0/5). Bilateral knee jerks, ankle jerks and plantar responses were absent. There was no response to pain in the lower extremities.

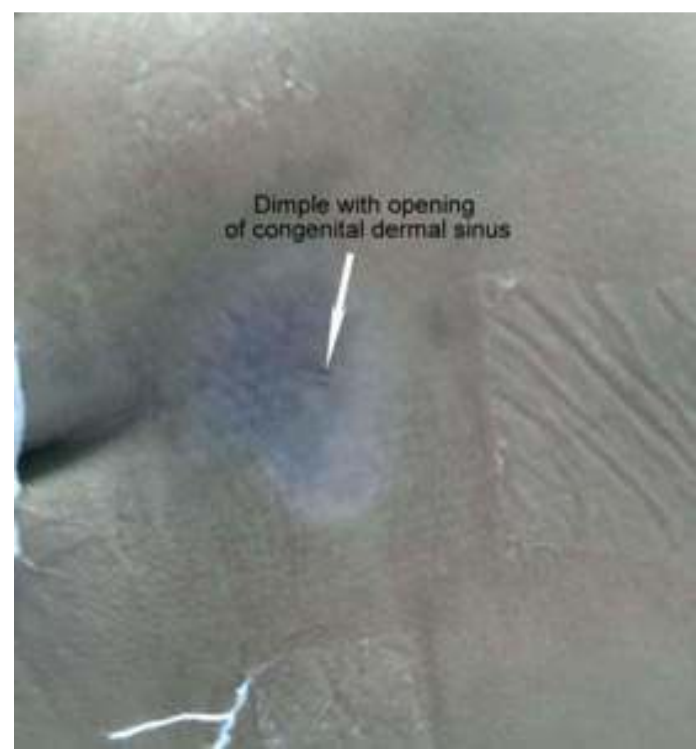

Figure 1: Neurocutaneous marker on the back with opening of congenital dermal sinus

Laboratory investigations showed leucocytosis with neutrophilia (White blood cell count $23,000 / \mu \mathrm{L}$ with $60 \%$ neutrophils) and elevated C-reactive protein $(22.5 \mathrm{mg} / \mathrm{L}$; normal $<5 \mathrm{mg} / \mathrm{L})$. X-ray of lumbosacral spine showed dorsolumbar scoliosis. Magnetic resonance imaging (MRI) of spine showed absent posterior elements at $\mathrm{S} 1$ and $\mathrm{S} 2$ with tethered cord and enlarged conus. There was an intramedullary abscess extending from D3 to S2 with a sinus tract extending to the skin from the thecal sac attached to the conus (Figure 2).

She underwent L3 to S2 laminoplasty with excision of the dermal sinus. The intramedullary abscess was evacuated and the tethered cord released (Figure 3). An epidermoid tumour associated with the CDS noticed intraoperatively was also excised (Figure 4). 

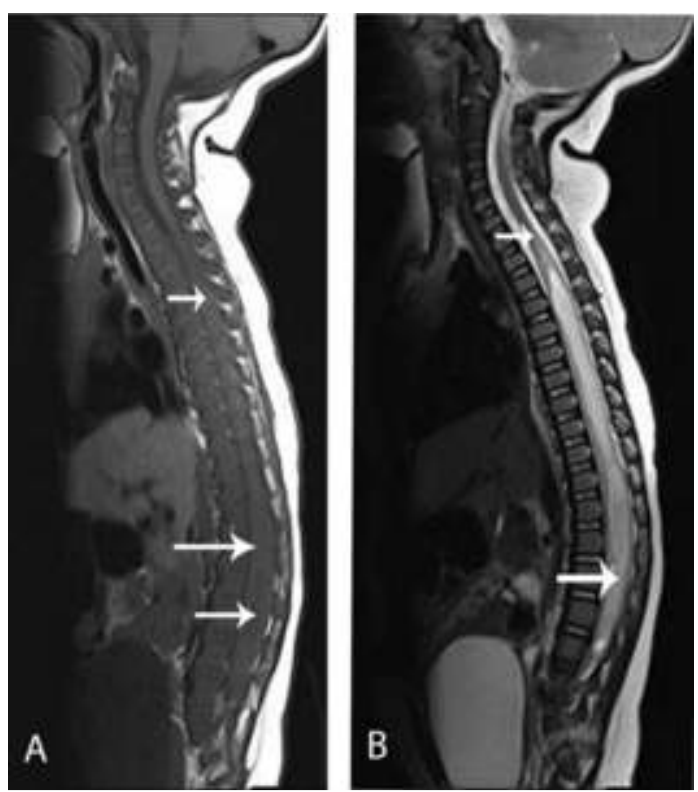

Figure 2:A. Sagittal T1WI shows diffuse cord expansion with a hypointense intramedullary lesion (white arrows). B. Corresponding sagittal T2WI whole spine reveals hyperintense lesion involving conus medullaris extending up to D3 level \& rostral thoracic cord oedema (white arrows)

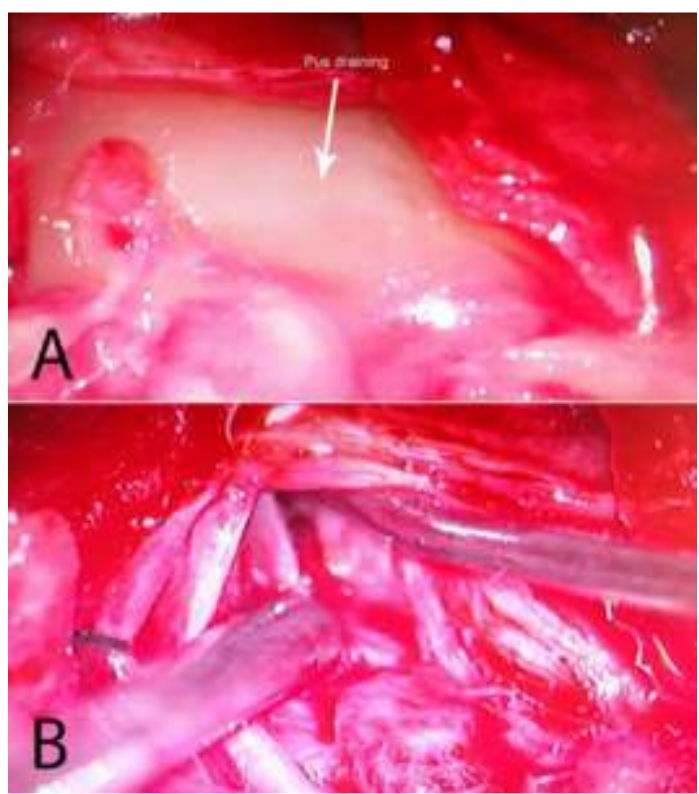

Figure 3:A. Pus draining out at surgery (white arrow). B. Abscess cavity

Gram staining of pus drained from the intramedullary abscess showed gram positive cocci with culture growing methicillin-resistant Staphylococcus aureus (MRSA). Histopathology confirmed an infected dermal sinus. She was treated with intravenous antibiotics and her neurological status started improving immediately after surgery. She started moving her right leg followed by her left lower limb and at the time of discharge she had only minimal weakness of both legs. At review after a week she had regained $4 / 5$ power of both lower limbs.

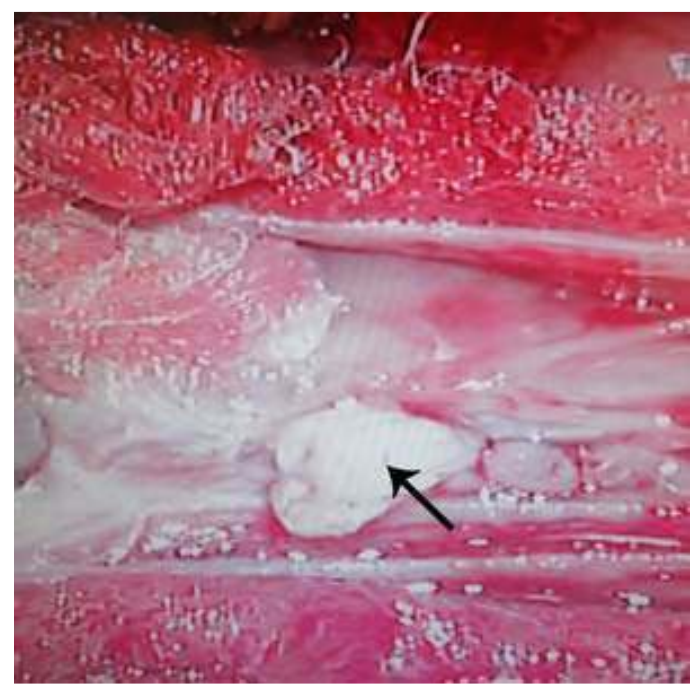

Figure 4: Intra operative picture of epidermoid (black arrow)

\section{Discussion}

Intramedullary spinal cord abscess (ISCA) is a rare suppurative infection of the CNS, especially in children $^{1,2}$.Congenital dermal sinus (CDS) is an uncommon form of spinal dysraphism, which if ignored can cause meningitis or infect the spaces around the spinal cord or lead to ISCA as has happened to our patient $t^{1,2}$. In all probability the clinicians consulted initially confused CDS with coccygeal pit, a benign and distinctly different entity occurring in approximately $4 \%$ of the general population ${ }^{3}$. CDSs are located above the gluteal cleft, have a cephalically oriented tract, and are often associated with cutaneous marker and intradural pathology while the latter is located within the gluteal cleft, are oriented caudally or straight down, and are not associated with intradural pathology ${ }^{3}$. CDS may be associated with inclusion tumours such as dermoid, epidermoid, teratoma, diastematomyelia and tethered cord ${ }^{3}$. Our patient had an epidermoid tumour associated with the tract which was detected only during surgery (Figure 4). It is documented in the literature that the associated tumours may be revealed by MRI or rarely only at the time of operation ${ }^{2,3}$.

Dermal sinus is the leading cause of ISCA in children with incidence varying from $25-75 \%^{1,2}$. Thoracolumbar region of spinal cord is the frequent site of involvement ${ }^{4,5}$. Chan et al reported that all cases of ISCA resulting from CDS had associated dorsal midline skin lesions ${ }^{1}$. Our patient also had the same but whether CDS with midline skin lesions have increased chance of developing ISCA 
is not clear ${ }^{1,4,5}$. Intramedullary spinal cord abscess may present at any age but children under the age of five are more likely to be affected ${ }^{2,5}$. Mean age of presentation has decreased over the years ${ }^{1,2}$. Patients with infections from contiguous spread are usually younger than those with infections secondary to other mechanisms ${ }^{1,2}$. Once infection develops in the spinal cord, it spreads longitudinally along the fibre tracts of the cord. The abscess starts in the grey matter and extends peripherally into the white matter ${ }^{4}$. The mean range of involvement is three to six levels and but holocord involvement has also been described ${ }^{2,4,5}$.

Clinical features depend on the location of abscess, age and time of presentation ${ }^{3,4,6}$.Average time from onset of symptoms to diagnosis ranges from $0-540$ days (mean 46 days) ${ }^{1,4}$. In a review of cases from 1875 - 2001, the single most sensitive and objective indicator of intramedullary spinal cord abscess in children was paralysis, which was documented in $58 \%$ patients ${ }^{6}$. Patients usually present with motor impairment followed by sensory loss, sphincter disturbances and dorsal pain ${ }^{4}$. Fever may be absent in two-thirds of patients ${ }^{1,6}$. The commonest causative organism of an abscess associated with dermal sinus is Staphylococcus aureus ${ }^{5,6}$.

MRI with gadolinium enhancement is the imaging modality of choice for analysing the location and extent of the spinal cord lesions ${ }^{1}$. MRI features of abscess formation include increased signal intensity on sagittal T2-weighted images and central lowsignal-intensity lesions with peripheral gadolinium enhancement on sagittal T1-weighted images ${ }^{1}$. The radiological findings may be difficult to differentiate from an intramedullary tumour as with our patient. Diffusion-weighted imaging (DWI) has been recommended as a more sensitive and specific method for differentiating intra-spinal abscesses from cystic or necrotic tumours ${ }^{7,8}$.

Treatment involves broad-spectrum antimicrobial therapy for 6-8weeks, steroids, limited laminectomy and myelotomy with copious irrigation with normal saline and simultaneous correction of any concomitant abnormalities ${ }^{6}$. Early recognition and systematic management of CDS is extremely important because many children may have serious neurological deficits at initial presentation, the extent and severity of which will worsen with increasing age ${ }^{3}$.

All newborns and infants should be examined for dermal sinus and other stigmata of spinal dysraphism. Prompt imaging should be done in all cases detected especially if it is above gluteal fold, greater than $5 \mathrm{~mm}$ in diameter and more than $2.5 \mathrm{~cm}$ from the anal opening. In CDS surgical exploration is advised even if the MRI is normal as there is a chance that tract may be missed if its trajectory is out of the imaging plane ${ }^{3}$. Midline neuro-cutaneous marker in the back is almost always pathological requiring detailed radiological and neurosurgical evaluation.

\section{References}

1. Chan CT, Gold WL. Intramedullary abscess of the spinal cord in the antibiotic era: clinical features, microbial aetiologies, trends in pathogenesis, and outcomes. Clinical Infectious Diseases 1998; 27(3):619-26. http://dx.doi.org/10.1086/514699

2. Al Barbarawi M, Khriesat W, Qudsieh S, Qudsieh H, Loai AA. Management of intramedullary spinal cord abscess: experience with four cases, pathophysiology and outcomes. European Spine Journal 2009; 18(5):710-7. http://dx.doi.org/10.1007/s00586-0090885-0

PMid: 19172309 PMCid: PMC3234004

3. Ackerman LL, Menezes AH. Spinal congenital dermal sinuses: a 30-year experience. Pediatrics 2003; 112: 3(1) 641-7.

4. Kumar R, Arora R. Pediatric spinal infections. Indian Journal of Neurosurgery 2014; 3:75-85. http://dx.doi.org/10.4103/22779167.13889 1

5. Desai KI, Muzumdar DP, Goel A. Holocord intramedullary abscess: an unusual case with review of literature. Spinal Cord 1999; 37(12):866-70. http://dx.doi.org/10.1038/sj.sc.3100930 PMid: 10602531

6. Volkan ETUS. Intramedullary spinal cord abscesses in children: Review. Journal of Neurological Science 2011; 28(4):651-61.

7. Aggarwal M, Aggarwal KC, Aggarwal A. Intramedullary spinal cord abscess masquerading as spinal tumour. Indian Pediatrics 2011; 48(12):973-4.

PMid: 22253156

8. Dörflinger-Hejlek E, Kirsch EC, Reiter H, Opravil M, Kaim AH. DiffusionWeighted MR imaging of intramedullary spinal cord abscess. American Journal of Neuroradiology 2010; 31(9):1651-2. http://dx.doi.org/10.3174/ajnr.A1912 PMid: 20019101 